\title{
A história recente do Futsal na UFSM no contexto do ensino, da pesquisa e da extensão
}

\author{
The recent history of Futsal at UFSM in the context of teaching, research and extension
}
La historia reciente del Fútbol Sala en la UFSM en el contexto de la docencia, la investigación y la extensión

Braulio da Silva Machado, ${ }^{\text {I }}$ Antonio Guilherme Schmitz Filho ${ }^{\text {II }}$, Gabriel Ivan Pranke ${ }^{\mathrm{III}}$

\begin{abstract}
Resumo
O presente artigo busca contribuir na composição do Dossiê Temático: CEFD/UFSM 50 anos: histórico e transformações na Educação Física a partir da estruturação histórica da equipe representativa da UFSM na modalidade de Futsal ao longo dos últimos 15 (quinze) anos. Com destaque na iniciativa dos acadêmicos de Educação Física no ano de 2005, o texto introduz o histórico esportivo da equipe e recobre as articulações institucionais estabelecidas sob o registro e reformulação de projetos de extensão coordenados por diferentes docentes do Departamento de Desportos Coletivos do CEFD. A indicação dos objetivos recondicionados na última atualização do projeto contextualiza a atual etapa de engendramentos entre ensino, pesquisa e extensão que se articulam na perspectiva do desenvolvimento esportivo a partir da UFSM. Por fim, a apresentação das considerações finais abrange as expectativas de médio e longo prazo para diferentes frentes de atuação que o Futsal abrange no contexto institucional e regional.
\end{abstract}

Palavras-chave: Futsal; Ensino; Pesquisa; Extensão; História

\begin{abstract}
This article seeks to contribute to the composition of the Thematic Dossier: CEFD/UFSM 50 years: history and transformations in Physical Education from the historical structuring of the representative team of UFSM in the Futsal modality over the last 15 (fifteen) years. With emphasis on the initiative of Physical Education students in 2005, the text introduces the team's sporting history and covers the institutional articulations established under the registration and reformulation of extension projects coordinated by different professors of the Collective Sports Department of CEFD. The indication of the refurbished objectives in the last update of the project contextualizes the current stage of engenderments between teaching, research and extension that are articulated in the perspective of sports development from UFSM. Finally, the presentation of the final considerations covers the medium and long term expectations for different fronts of action that Futsal covers in the institutional and regional context.
\end{abstract}

Keywords: Futsal; Teaching; Research; Extension; History

\footnotetext{
${ }^{\text {I } U n i v e r s i d a d e ~ F e d e r a l ~ d e ~ S a n t a ~ M a r i a ~-~ U F S M ~-~ E n d e r e c ̧ o: ~ A v . ~ R o r a i m a, ~ n u ́ m e r o ~ 1000, ~ B a i r r o: ~ C a m o b i, ~ P r e ́ d i o ~ 51, ~ S a n t a ~ M a r i a, ~ R S . ~ C E P: ~} 97105-900$ - email: brauliomachado.fut@gmail.com

II Universidade Federal de Santa Maria - UFSM - e-mail: brauliomachado.fut@gmail.com

III Universidade Federal de Santa Maria - UFSM - e-mail: pranke.cefd@ gmail.com
} 


\section{Resumen}

Este artículo busca contribuir a la composición del Dossier Temático: CEFD/UFSM 50 años: historia y transformaciones en Educación Física a partir de la estructuración histórica del equipo representativo de la UFSM en la modalidad de Fútbol Sala en los últimos 15 (quince) años. Con énfasis en la iniciativa de los estudiantes de Educación Física en 2005, el texto introduce la historia deportiva del equipo y cubre las articulaciones institucionales establecidas bajo el registro y reformulación de proyectos de extensión coordinados por diferentes profesores del Departamento de Deportes Colectivos del CEFD. La indicación de los objetivos reacondicionados en la última actualización del proyecto contextualiza la etapa actual de engendros entre enseñanza, investigación y extensión que se articulan en la perspectiva del desarrollo deportivo desde la UFSM. Finalmente, la presentación de las consideraciones finales cubre las expectativas de mediano y largo plazo para los diferentes frentes de acción que cubre el Futsal en el contexto institucional y regional.

Palabras clave: Fútbol sala; Enseñanza; Investigación; Extensión; Historia

\section{Introdução}

A equipe de Futsal da UFSM, que completa em 2020 seu décimo quinto ano de atividade apresenta como base de sustentação em seu histórico esportivo o envolvimento acadêmico de seus integrantes desde o ano de 2005, o que condiciona o encaminhamento do presente texto como proposta para a composição do Dossiê Temático: CEFD/UFSM 50 anos: histórico e transformações na Educação Física.

O projeto teve origem na iniciativa dos acadêmicos do curso de Educação Física, que contaram na época com o apoio do Professor Cyro Knackfuss para oficializar, junto ao Gabinete de Projetos do Centro de Educação Física e Desportos (CEFD), os primeiros passos desta jornada esportiva através do projeto de extensão Futsal no CEFD (registro GAP/CEFD n 018160). Em seguida, no ano de 2006, com a criação do Núcleo Universitário de Esportes (NUE), o projeto passou a integrar o programa de extensão Esporte, Cultura e Jornalismo: Esboços para ações a partir da UFSM (registro GAP/CEFD n 019470), sob a tutela do Professor Antonio Guilherme Schmitz Filho.

As configurações iniciais incrementaram de forma preliminar e consistente, com referência em elementos constituintes da tese de doutorado de Schmitz Filho (2005), a contextualização acadêmica entre o ensino, a pesquisa e a extensão. De tal forma que os treinamentos da equipe de Futsal, que posteriormente integraram o projeto de extensão Departamento Experimental de Futebol: Equipes Laboratório (registro GAP/CEFD n 029292) ocorreram em consonância à Disciplina Complementar de Graduação (DCG) denominada de Aprofundamento nos Esportes I - DEC 1036 (com ênfase no Futsal), oferecida pelo Departamento de Desportos Coletivos (DDC) do Centro de Educação Física e Desportos (CEFD). 
Cabe destacar que a estruturação acadêmica citada, até o ano de 2018, esteve inserida no organograma do Laboratório de Análise dos Cenários Esportivos na Mídia (LACEM), que desde o início de suas atividades (agosto de 2010), investiga a compreensão do jogo em diferentes modalidades esportivas a partir de contextos midiáticos e da sua ressignificação para o ensino esportivo. Nos 15 (quinze) anos de existência, a equipe participou de competições como os Jogos Universitários de Santa Maria (JUSM), Jogos Universitários Gaúchos (JUGs), Jogos Universitários Brasileiros (JUBs) e Jogos Universitários do Mercosul, além de competições amadoras na cidade de Santa Maria.

Desde o ano de 2016, após a reformulação do projeto e registro sob o título UFSM na Série Bronze do Futsal Gaúcho (registro GAP/CEFD n 041680), além do circuito esportivo universitário, a equipe de Futsal da UFSM passou a integrar, via filiação, a Federação Gaúcha de Futsal (FGFS), participando do Campeonato Gaúcho entre os anos de 2016 e 2018, pela Série Bronze e, a partir de 2019, pela Série Ouro da competição. Entre os ano de 2017 e 2019, a UFSM também participou do certame na categoria sub-20.

Para além das conquistas esportivas alcançadas no período, que somam um bicampeonato dos Jogos Universitários Gaúchos e um tricampeonato dos Jogos Universitários do Mercosul, ganham destaque as contribuições acadêmicas que o projeto proporciona a diferentes áreas do conhecimento. A equipe serviu e serve como campo de estágio para diversos acadêmicos do curso de Educação Física, e constantemente é utilizada como objeto de estudo para diversas pesquisas científicas nos campos da pedagogia do esporte, jornalismo esportivo, publicidade e propaganda, fisiologia do exercício, biomecânica, cineantropometria, fisioterapia, psicologia, medicina, análise de desempenho e outras áreas.

O Futsal é uma modalidade que possui centralidade na cultura esportiva gaúcha. Sua abrangência junto ao ensino escolar é muito grande. As crianças acorrem à prática quase que de forma natural. Neste contexto, e a partir de uma projeção da participação na Série Bronze do Futsal Gaúcho desde o ano de 2016, teve início a articulação no campo acadêmico de discussões sobre o ensino esportivo com densidade e protagonismo voltados a esta realidade.

Os requisitos esportivos passados de uma geração para a outra são os grandes constituintes de uma dada cultura. Eles não devem ser esquecidos ou deixados à margem em um processo de desenvolvimento. Decorre do envolvimento com o aspecto cultural a necessidade de se relacionar o conhecimento pregresso com a efetiva atuação prática. Portanto, o campo acadêmico torna-se promissor para a exploração contextualizada do fenômeno esportivo na sociedade.A seguir são apresentados os objetivos do projeto a partir de sua reformulação no ano de 2016, que conta com ações de ensino, pesquisa e extensão dentro da UFSM e na comunidade acadêmica em geral. 


\section{Objetivos}

Participar do Campeonato Estadual de Futsal;

Estimular a formação de uma cultura esportiva regional a partir do ensino, da pesquisa e da extensão na UFSM;

Desenvolver uma metodologia de ensino para o Futsal através da aplicação de uma proposta para crianças e jovens;

Ampliar a execução de projetos de iniciação científica, trabalhos acadêmicos de conclusão de curso na graduação e pós-graduação lastreado em Grupo de Pesquisa específico de Futsal junto à Base LATTES;

Sedimentar o aprofundamento esportivo a partir da graduação no Curso de Educação Física da UFSM aplicado a situações reais do ensino e do treinamento esportivo;

Aprimorar o envolvimento da comunidade acadêmica com a prática do Futsal;

Promover eventos (seminários e congressos) relacionados com a temática esportiva envolvendo a modalidade.

\section{Descrição do Projeto}

\subsection{Situação Atual}

Os treinamentos da equipe de Futsal da UFSM acontecem no Ginásio Didático III do CEFD, todas terças, e quintas-feiras das $18 \mathrm{~h} 30 \mathrm{~m}$ até $20 \mathrm{~h} 30 \mathrm{~m}$, e nas quartas-feiras das 20h30m até 22h30m. Em consonância é desenvolvida a DCG de Aprofundamento nos Esportes (com ênfase na modalidade de futsal), o que gera uma relação direta para os acadêmicos do Curso de Educação Física no que diz respeito a uma realidade aplicada para a prática sistemática de treinamento desportivo.

Os acadêmicos matriculados na disciplina têm à disposição a oportunidade de acompanhar e participar efetivamente dos processos de planejamento, treinamento e produção de conhecimento acerca da modalidade de Futsal, compartilhando informações e conteúdos com integrantes da comissão técnica da equipe. Desde o primeiro semestre letivo de 2015 o grupo se envolveu na preparação e participação da equipe nos Jogos Universitários Gaúchos, nos Jogos Universitários do Mercosul e posteriormente nos Campeonatos Estaduais, nas Séries Bronze, Ouro e na categoria Sub-20.

A partir do ano de 2018 o projeto passou a ser coordenado pelo Professor Gabriel Ivan Pranke, que estabeleceu um período de imersão, durante o início do ano letivo (Semana de Imersão ao Futsal 
UFSM), em que todo o grupo de acadêmicos e integrantes externos que compõe a comissão técnica se reúne para discussões acerca das diferentes áreas que envolvem o processo de desenvolvimento esportivo.

O período é marcado por palestras de profissionais atuantes na modalidade e oriundos de diversas áreas profissionais. Entre outras coisas, todo o planejamento anual é realizado nesta etapa, o que inclui avaliações físicas e periodização de treinamentos, prevenção e tratamento de lesões, orçamentos, captação de recursos e logística para as competições disputadas, planejamento de seminários, congressos e dos processos de iniciação esportiva em inserções realizadas em escolas parceiras da rede pública, entre outras frentes de atuação sobre as quais o projeto se debruça.

Com a suspensão das atividades presenciais na instituição e da perspectiva de início das competições esportivas para o ano de 2020 por conta da pandemia do COVID-19, o período de preparação da comissão técnica e planejamento das atividades se estendeu e vem sendo realizado de forma remota ao longo do semestre. Entre as ações, destaca-se um circuito de aulas abertas oferecido à comunidade acadêmica, disponível nas referências deste artigo, que contou com a colaboração de diversos profissionais e foi apresentado pela plataforma www.youtube.com, na página Oficial da UFSM Futsal $^{1}$.

Atualmente a expectativa gira em torno da retomada das atividades presenciais na UFSM para o posterior planejamento das ações necessárias à retomada de treinamentos e competições, orientados por protocolos de segurança que visem a acomodação de todos os integrantes do projeto em suas respectivas funções.

\subsection{Perspectivas}

A expectativa de atuação da equipe de Futsal da UFSM gira em torno da participação no Campeonato Estadual de Futsal - Série Ouro. A participação na competição tem potencializado as possibilidades de expansão das contribuições acadêmicas e esportivas que o projeto já oferecia desde 2006.

A simples perspectiva de aproximação entre teoria e prática num ambiente que ao mesmo tempo se configura como profissional e acadêmico remete a uma realidade que até o momento se reveste de grande significância, tanto para a academia como um todo, como também para a própria modalidade, naquilo que diz respeito ao contexto regional que envolve a cidade de Santa Maria.

${ }^{1}$ https://www.youtube.com/channel/UCEdtEclnn7pDgZaiaVT2IuA 
O movimento de participação em evento de porte regional, por si só pressupõe a necessidade de envolvimento de campos do conhecimento que se sobrepõem no que se refere ao desenvolvimento esportivo, como a Educação Física, a Pedagogia, a Fisioterapia, a Psicologia, a Nutrição, a Medicina, o Jornalismo, a Administração, o Direito, entre outros. Neste sentido, uma série de relações encontram-se presentes nas proposições do projeto. São possibilidades multidisciplinares que se desdobram em diversas ações que o projeto aponta.

As perspectivas tornam-se relevantes, na medida em que a UFSM assume protagonismo através da representatividade que a equipe de Futsal oferece, uma vez que o nome da Instituição passa a ser vinculado em todo o estado a uma ideia inédita também entre Instituições Federais de Ensino.

\subsubsection{No ensino}

A DCG com ênfase no futsal ingressou na grade curricular do Curso de Educação Física como uma excelente possibilidade de ampliação estrutural para o ensino esportivo a partir do CEFD, uma vez que a modalidade é amplamente difundida no Rio grande do Sul e junto ao ambiente escolar, ampliando o campo de experimentação e trabalho na área da formação esportiva.

Mesmo com um status vantajoso em relação à cultura esportiva no Brasil, o futebol, assim como o futsal, segundo Schmitz Filho (1999) e Machado (2012), carece de uma ressignificação e discussão acerca do seu contexto epistemológico, cultural e prático, a partir dos bancos acadêmicos. As diversas estruturas esportivas existentes caracterizam concepções que merecem revisão e análise abrangentes, no sentido do estabelecimento de novas abordagens e entendimentos para aquilo que se produz no campo esportivo.

Se tomado como um dos pontos de partida, o ambiente escolar, espaço em que a paixão pelo futebol é em muitas ocasiões traduzida nas aulas de Educação Física através do jogo de futsal, considerando-se os espaços disponíveis e a facilidade de organização do jogo (ambiente reduzido), existe aí uma possibilidade imediata e direta de interação com tal manifestação a partir da DCG citada.

Outro aspecto fundamental no que se refere ao ensino esportivo para crianças e jovens a partir da disciplina de aprofundamento esportivo é a criação de atividades de iniciação para crianças oriundas da rede pública de educação. Uma questão represada desde muito tempo e que, com certeza, merece o devido cuidado e zelo para a sua manutenção (planejamento adequado para a garantia de oferta e continuidade).

No que tange a relação com a grade curricular, as possibilidades de estágios oferecidas junto à DCG, composta pela equipe de futsal são garantidas no próprio ambiente formativo (CEFD), o que 
colabora de forma efetiva para o acompanhamento dos mesmos, bem como na discussão e planejamento das necessidades relacionadas com a evolução dos acadêmicos envolvidos.

\subsubsection{Na pesquisa}

O LACEM, desde a sua criação em agosto de 2010 desenvolve investigações acerca da influência da midiatização esportiva para a compreensão de jogo em diferentes modalidades e consequentemente os impactos causados nos processos de ensino e treinamento esportivo, como nos casos dos estudos de Santos (2020), Piegas Junior (2020), Machado (2019), , que se debruçaram especificamente sobre as modalidades de Futebol e Futsal.

A participação no Campeonato Estadual de Futsal - Série Ouro amplia de forma significativa as possibilidades de investigações desenvolvidas através de projetos de pesquisa, trabalhos de conclusão de curso na graduação e pós-graduação, assim como na sustentação de um grupo de pesquisa especificamente voltado para o futsal na Base Lattes. Cada movimento neste sentido possibilita o estabelecimento de investigações científicas com diferentes abordagens através dos laboratórios de pesquisa do CEFD.

O processo de preparação para uma competição de longo prazo como é o caso do estadual de Futsal, traz impactos acadêmicos muito significativos na área da pesquisa científica. As variáveis de amostra para qualquer área do conhecimento que se interesse em investigar o fenômeno esportivo se multiplicam na medida em que as ações descritas a seguir se sistematizam no desenvolvimento do projeto.

Obviamente que a aproximação dos diferentes campos do conhecimento para investigação científica encontra-se condicionada ao potencial de contribuição apresentado pelos respectivos projetos de pesquisa (devidamente registrados) em relação ao desenvolvimento da modalidade nos diferentes níveis de ensino e treinamento.

\subsubsection{Na extensão}

A participação da Equipe de Futsal da UFSM no Campeonato Estadual de Futsal - Série Ouro impôs uma expansão ao projeto de extensão Departamento Experimental de Futebol: Equipes Laboratório. Neste sentido, e considerando-se a vinculação ao programa de extensão intitulado Esporte, Cultura e Jornalismo: Esboços para ações a partir da UFSM surgiram novas oportunidades de atuação para as diversas frentes extensionistas vigentes. 
A constituição de uma comissão técnica e de um grupo de atletas alinhados academicamente com o projeto tornou-se ponto importante para a sustentação da proposta (relação efetiva com o ensino e o treinamento esportivo). A dimensão estimada para os objetivos acadêmicos passa pela atuação em diferentes frentes (ensino, pesquisa e extensão) que são perenemente sobrepostas no desenvolvimento do trabalho.

O propósito principal é que além dos treinamentos da equipe, realizados durante as aulas da DCG de Futsal, a comissão técnica e o grupo de atletas se envolvam também com outros projetos oriundos do Programa de Extensão citado anteriormente. A iniciativa pretende mobilizar a instituição a partir do futsal para o fortalecimento de uma cultura esportiva com ofertas diversificadas e constantes.

Os integrantes da equipe participarão da organização das atividades. A ideia é que os eventos mobilizem o maior número de pessoas possível e chamem atenção para o sentido esportivo que o movimento pretende estabelecer (a ampliação regular e consequente de oportunidades para práticas esportivas).

Outra perspectiva extensionista que vem sendo desenvolvida diz respeito à iniciação esportiva voltada ao futsal na UFSM. Em um primeiro momento o foco de oferta de atividades está concentrado na rede pública de ensino, vinculando sua expansão a cada semestre letivo em conformidade à demanda.

A mobilização para a participação no campeonato estadual também se sustenta com o foco na iniciação esportiva. É no desenvolvimento esportivo que se justifica a idealização do projeto a longo prazo. Para tanto a iniciação esportiva junto à modalidade na UFSM, abrange a participação dos integrantes da comissão técnica e serve ao desenvolvimento de uma proposta metodológica renovada para o ensino esportivo.

A metodologia de uma maneira geral, aponta para uma preocupação constante relacionada com a compreensão de jogo, em alinhamento com propostas recolhidas na obra de Mahlo (1997), bem como uma prática de ensino, treinamento e competições ajustadas especificamente para as faixas etárias atendidas no projeto, no intuito de envolver inúmeros aspectos do desenvolvimento dos jovens e das crianças.

A iniciação esportiva com base no futsal na UFSM, através de um Projeto de Extensão se relaciona diretamente com o ensino e a pesquisa, assim como todas as possibilidades de atuação mencionadas na proposta, o que configura a possibilidade de estabelecimento de um maior número de projetos integrados (ensino, pesquisa e extensão).

E por último, a organização de seminários e congressos para tratar das mais variadas temáticas que cercam a modalidade de futsal preenche o universo de ações de extensão pretendidas na idealização do projeto para o Futsal da UFSM. A ideia é oferecer aos acadêmicos de diferentes cursos da instituição e 
aos integrantes das comissões técnicas das equipes participantes das competições organizadas pela Federação Gaúcha de Futsal (FGFS), a oportunidade de atualização e discussão de pormenores que cercam o desenvolvimento da modalidade. É esse o caso do $1^{\circ}$ Congresso de Futsal da UFSM que aconteceria no primeiro semestre de 2020, mas que foi adiado em virtude da pandemia.

\section{Considerações Finais}

Na perspectiva de conclusão do artigo, no contexto de médio e longo prazo, um processo de avaliação constante de resultados torna-se imprescindível para a adequação dos objetivos do projeto. Sobretudo, aqueles que dizem respeito ao campo acadêmico. O ponto fundamental para sustentação das ações é a formação de uma cultura de prática da modalidade como apoio ao desenvolvimento esportivo.

A participação nas diversas competições oferece importante suporte para a manutenção do envolvimento da comunidade acadêmica com a modalidade. A sustentação da equipe de Futsal da UFSM propicia o recolhimento de elementos epistemológicos fundamentais ligados à realidade que cerca o esporte na atualidade. E indica processos necessários para incrementar as ações de ensino, pesquisa e extensão em desenvolvimento dentro da UFSM. Novos projetos, ações e atividades de extensão, como a iniciação esportiva, o circuito de aulas abertas, seminários e congressos sobre Futsal na UFSM adquirem protagonismo na processualidade da proposta.

A estimativa é de que o envolvimento com a modalidade cresça como cultura esportiva, e que dessa realidade surjam novas frentes de atuação para dar conta de um desenvolvimento esportivo regular. Embora muitas pessoas pratiquem a modalidade, existe um envolvimento acadêmico com o futsal dentro da UFSM ainda muito restritivo aos acadêmicos do curso de Educação Física, envolvidos com a equipe.

Nos anos anteriores ao recondicionamento do projeto esse envolvimento oscilava de acordo com a proximidade das competições disputadas a cada semestre. A idealização da participação no Campeonato Estadual de Futsal pressupõe um envolvimento que além de mais abrangente, do ponto de vista das diversas potencialidades acadêmicas, se torna constante devido ao fato da competição se estender ao longo de todo o ano letivo.

Portanto, a médio e longo prazo é perfeitamente viável garantir uma qualificação acadêmica e profissional ainda maior para os participantes em qualquer uma das frentes apresentadas no projeto. A proposta é de envolvimento prático e real com os pormenores do desenvolvimento esportivo em diferentes níveis, o que eleva o potencial formativo para os diversos campos do conhecimento. 
Vale ressaltar ainda, que não só a graduação ganha com o processo. A pós-graduação, que por sua vez tem a necessidade constante de qualificar a produção científica para sua manutenção, tem neste caso, a perspectiva de desenvolver estudos em diferentes cursos e linhas de pesquisa. A título de exemplo, existem neste momento três estudos em estreita relação com a realidade atual (trabalho remoto e educação à distância), envolvendo a modalidade de Futsal, em desenvolvimento ao nível de Mestrado e sob orientação do Professor Antonio Guilherme Schmitz Filho, no Programa de Pós-Graduação em Tecnologias Educacionais em Rede da UFSM.

\section{Referências}

ALVES, Gabriel de Moura Nardes. Protagonismo midiático esportivo: relações com a Educação Física escolar. 2018. Trabalho de Conclusão de Curso (Especialização em Educação Física Escolar)Universidade Federal de Santa Maria, Santa Maria, 2018. Disponível em: <https://repositorio.ufsm.br/handle/1/13811>. Acesso em: 13 ago. 2020.

BElinASSO, Bruna. A Copa das Confederações de 2013: predições, surpresas e realidade. 2015. Trabalho de Conclusão de Curso (Especialização em Educação Física Escolar)-Universidade Federal de Santa Maria, Santa Maria, 2015. Disponível em: 〈https://repositorio.ufsm.br/handle/1/14790〉. Acesso em: 13 ago. 2020.

BETTEGA, Otávio Baggiotto. A trajetória do Futsal santamariense na década de 1990: uma proposta de compreensão para o desenvolvimento tático a partir de perspectivas do jornal A Razão. 2011. Trabalho de Conclusão de Curso (Especialização em Atividade Física, Desempenho Motor e Saúde)-Universidade Federal de Santa Maria, Santa Maria, 2011. Disponível em: 〈https://repositorio.ufsm.br/handle/1/13701〉. Acesso em: 13 ago. 2020.

BITENCOURT, William Daniel. Uma discussão sobre a arbitragem e sua utilização no contexto esportivo escolar. 2016. Trabalho de Conclusão de Curso (Especialização em Educação Física Escolar)Universidade Federal de Santa Maria, Santa Maria, 2016. Disponível em: <https://repositorio.ufsm.br/handle/1/15002>. Acesso em: 13 ago. 2020.

BRANDÃO, Mariane da Silva. O feedback no ensino esportivo: conceituações e apontamentos para a sua utilização. 2012. Trabalho de Conclusão de Curso (Especialização em Atividade Física, Desempenho Motor e Saúde)-Universidade Federal de Santa Maria, Santa Maria, 2012. Disponível em: <https://repositorio.ufsm.br/handle/1/13745 >. Acesso em: 13 ago. 2020.

CAIRRÃO, Marcos Roberto. A midiatização da técnica no Futsal como suporte para o desenvolvimento de produtos educacionais. 2018. Trabalho de Conclusão de Curso (Especialização em Educação Física Escolar)-Universidade Federal de Santa Maria, Santa Maria, 2018. Disponível em: <https://repositorio.ufsm.br/handle/1/13493>. Acesso em: 13 ago. 2020.

CERATTI, Daniella Araújo. Socialização primária: um ensaio sobre oportunidades de movimento. 2017. Trabalho de Conclusão de Curso (Especialização em Educação Física Escolar)-Universidade Federal de Santa Maria, Santa Maria, 2017. Disponível em: <https://repositorio.ufsm.br/handle/1/131236>. Acesso em: 13 ago. 2020. 
DARONCH, Heitor dos Santos. Notoriedade no Futebol brasileiro: o caso Neymar nos Jogos Olímpicos de Londres 2012, sobre a ótica da plataforma virtual www.globoesporte.com. 2013. Trabalho de Conclusão de Curso (Especialização em Atividade Física, Desempenho Motor e Saúde)-Universidade Federal de Santa Maria, Santa Maria, 2013. Disponível em: 〈https://repositorio.ufsm.br/handle/1/186〉. Acesso em: 13 ago. 2020.

GASPARETTO, Giuliano Rossi. Algumas premissas sobre a compreensão do jogo de Futsal. 2010. Trabalho de Conclusão de Curso (Especialização em Atividade Física, Desempenho Motor e Saúde)Universidade Federal de Santa Maria, Santa Maria, 2010. Disponível em: <https://repositorio.ufsm.br/handle/1/1959>. Acesso em: 13 ago. 2020.

KAUFMANN, Mateus. O ensino esportivo e a necessidade de análise da influência midiática: considerações às apreciações e análises do ataque no Futsal. 2010. Trabalho de Conclusão de Curso (Especialização em Atividade Física, Desempenho Motor e Saúde)-Universidade Federal de Santa Maria, Santa Maria, 2010. Disponível em: <https://repositorio.ufsm.br/handle/1/2429>. Acesso em: 13 ago. 2020.

LEONEL, Rodrigo de Oliveira. Ensino esportivo do Futebol e EAD: ensaios adaptativos na plataforma MOODLE. 2012. Trabalho de Conclusão de Curso (Especialização em Atividade Física, Desempenho Motor e Saúde)-Universidade Federal de Santa Maria, Santa Maria, 2012. Disponível em: <https://repositorio.ufsm.br/handle/1/2680>. Acesso em: 13 ago. 2020.

MACHADO, Braulio da Silva. Jornalismo esportivo na copa do mundo de Futsal FIFA 2008: proposições didáticas para o ensino do Futsal. 2012. 72 p. Monografia (Especialização em Atividade Física, Desempenho Motor e Saúde)-Universidade Federal de Santa Maria, Santa Maria, 2012. Disponível em: 〈https://repositorio.ufsm.br/handle/1/2959>. Acesso em: 13 ago. 2020.

MACHADO, Braulio da Silva; SCHMITZ FILHO, Antonio Guilherme; SANTOS, Darlei Comin dos. Perspectivas à prática esportiva escolar: considerações acerca das apreciações e análises sobre a técnica do Futsal midiatizado. In: Intercom: XV Congresso de Ciências da Comunicação na Região Sul, 15. 2010, São Leopoldo/RS. Anais... São Leopoldo/RS, 2010. Disponível em: <http://www.intercom.org.br/papers/regionais/sul2010/resumos/R20-1244-1.pdf>. Acesso em: 13 ago. 2020.

MACHADO, Jonatan De Agostini. Um relato de caso: experiência esportiva no Futsal, caminhos entre o Brasil e a Itália. 2019. Trabalho de Conclusão de Curso (Graduação em Educação Física - Licenciatura)Universidade Federal de Santa Maria, Santa Maria, 2019.

MAHLO, Fridrich. O acto táctico no jogo. 4. ed. Lisboa: Compendium,1997. 252 p.

MARQUES FILHO, Cesar Vieira. A Copa do Mundo de Futebol de 2014: cenários técnicos e táticos para uma compreensão de jogo. 2015. Trabalho de Conclusão de Curso (Especialização em Educação Física Escolar)-Universidade Federal de Santa Maria, Santa Maria, 2015. Disponível em: <https://repositorio.ufsm.br/handle/1/14953>. Acesso em: 13 ago. 2020.

MORAES, Cleiton Vinicius Braz. Aspectos etnometodológicos de uma proposta baseada na difusão esportiva. 2013. Trabalho de Conclusão de Curso (Especialização em Movimento Humano, Sociedade e 
Cultura)-Universidade Federal de Santa Maria, Santa Maria, 2013. Disponível em: <https://repositorio.ufsm.br/handle/1/93 >. Acesso em: 13 ago. 2020.

MORAES, Leonardo Braz. A seleção brasileira na Copa do Mundo de Futebol 2014: experiências radiofônicas na articulação de perspectivas didáticas para o esporte escolar. 2014. Trabalho de Conclusão de Curso (Especialização em Educação Física Escolar)-Universidade Federal de Santa Maria, Santa Maria, 2014. Disponível em: <https://repositorio.ufsm.br/handle/1/15623>. Acesso em: 13 ago. 2020.

PIEGAS JUNIOR, Carlos Ivan Ferraz. Apreciações de tática existentes no site esportivo globoesporte.com: uma proposta de análise para utilização no ensino esportivo. 2020. Trabalho de Conclusão de Curso (Especialização em Educação Física Escolar)-Universidade Federal de Santa Maria, Santa Maria, 2020.

PINHEIRO, Luis Eduardo Baldiatti. Jogo de pivô: o ensino esportivo na escola com referência na midiatização do jogo. 2018. Trabalho de Conclusão de Curso (Especialização em Educação Física Escolar)-Universidade Federal de Santa Maria, Santa Maria, 2018. Disponível em: <https://repositorio.ufsm.br/handle/1/15204>. Acesso em: 13 ago. 2020.

PRESTES, Marcelo Freitas. Atributos para a análise do regionalismo junto ao jornalismo esportivo brasileiro. 2013. Trabalho de Conclusão de Curso (Especialização em Atividade Física Desempenho Motor e Saúde)-Universidade Federal de Santa Maria, Santa Maria, 2013. Disponível em: <https://repositorio.ufsm.br/handle/1/265>. Acesso em: 13 ago. 2020.

SANTOS, Bernardo Carbone dos. A utilização do brincar como elemento pedagógico no ensino do Futsal no âmbito escolar. 2020. Trabalho de Conclusão de Curso (Especialização em Educação Física Escolar)-Universidade Federal de Santa Maria, Santa Maria, 2020.

SANTOS, Darlei Comin dos. A defesa no Futsal: o ensino esportivo e a necessidade de análise da influência midiática. 2010. Trabalho de Conclusão de Curso (Especialização em Atividade Física, Desempenho Motor e Saúde)-Universidade Federal de Santa Maria, Santa Maria, 2010. Disponível em: <https://repositorio.ufsm.br/handle/1/16513 >. Acesso em: 13 ago. 2020.

SANTOS, Flávia Fernandes. A mordida em frames: o caso do jogador Luís Suarez na Copa do Mundo de 2014. 2016. Trabalho de Conclusão de Curso (Especialização em Educação Física Escolar)Universidade Federal de Santa Maria, Santa Maria, 2016. Disponível em: <https://repositorio.ufsm.br/handle/1/15694>. Acesso em: 13 ago. 2020.

SCHMITZ FILHO, Antonio Guilherme. Jornalismo esportivo na copa de 1998: uma tentativa de análise crítica das críticas.1999. 195 p. Dissertação (Mestrado em Comunicação e Cultura)-Universidade Federal do Rio de Janeiro, Rio de Janeiro, 1999.

UFSM FUTSAL. Aula aberta: relação ataque-defesa na formação esportiva no Futsal. Youtube, 28 maio 2020. Disponível em: 〈https://www.youtube.com/watch?v=mumF67j5xWA\&t=2548s >. Acesso em: 13 ago. 2020.

Aula aberta: o papel do professor/treinador na formação esportiva no Futsal. Youtube, 04 jun. 2020. Disponível em: 〈https://www.youtube.com/watch?v=klGlPNYRoXU>. Acesso em: 13 ago. 2020. 
Aula aberta: análise de desempenho. Youtube, 02 jul. 2020. Disponível em: <https://www.youtube.com/watch?v=klGlPNYRoXU>. Acesso em: 13 ago. 2020.

Aula aberta: preparação de goleiros no Futsal. Youtube, 16 jul. 2020. Disponível em: <https://www.youtube.com/watch?v=klGlPNYRoXU>. Acesso em: 13 ago. 2020.

DISCIPLINA COMPLEMENTAR DE GRADUAÇÃO. Aprofundamento nos esportes. Departamento de Desportos Coletivos do Centro de Educação Física e Desportos da Universidade Federal de Santa Maria. DEC 1037. 2006. Disponível em: <https://www.ufsm.br/ementario/disciplinas/dec1037>. Acesso em: 13 ago. 2020.

PROJETO DE EXTENSÃO. Futsal no CEFD. Gabinete de Projetos do Centro de Educação Física e Desportos da Universidade Federal de Santa Maria. 2005 - 2008. Registro nº 018160. Disponível em: <https://portal.ufsm.br/projetos/publico/projetos/view.html?idProjeto=20857>. Acesso em: 13 ago. 2020.

. Esporte, cultura e jornalismo: esboços para ações a partir da UFSM. Gabinete de Projetos do Centro de Educação Física e Desportos da Universidade Federal de Santa Maria. 2006 - $2010.2 \quad$ Registro $\quad \mathrm{n}^{\mathrm{o}} \quad 019470 . \quad$ Disponível em: <https://portal.ufsm.br/projetos/publico/projetos/view.html?idProjeto=22371>. Acesso em: 13 ago. 2020.

Projeto departamento experimental de Futebol: equipes laboratório. Gabinete de Projetos do Centro de Educação Física e Desportos da Universidade Federal de Santa Maria. 2011 - 2016. $\quad$ Registro $\mathrm{n}^{\mathrm{o}} \quad 029292 . \quad$ Disponível em: <https://portal.ufsm.br/projetos/publico/projetos/view.html?idProjeto=34767>. Acesso em: 13 ago. 2020.

. Projeto UFSM na série bronze do Futsal gaúcho. Gabinete de Projetos do Centro de Educação Física e Desportos da Universidade Federal de Santa Maria. 2015 - 2021. Registro n ${ }^{\circ}$ 041680. Disponível em: <https://portal.ufsm.br/projetos/publico/projetos/view.html?idProjeto=51332>. Acesso em: 13 ago. 2020.

\section{Como citar este artigo}

MACHADO, B. S.; SCHMITZ FILHO, A. G.; PRANKE, G. I. A A história recente do Futsal na UFSM no contexto do ensino, da pesquisa e da extensão. Revista Kinesis, Santa Maria, Dossiê CEFD 50 anos, p.01-13, 2020.

* O presente trabalho não contou com apoio financeiro de nenhuma natureza para sua realização. 